Macedonian Pharmaceutical Bulletin, 66 (Suppl 1) 159 - 160 (2020)

Online ISSN 1857 - 8969

UDC: 615.014.4:615.015.16

DOI: 10.33320/maced.pharm.bull.2020.66.03.079

Short communication

\title{
Risk assessment on temperature and relative humidity deviation during on-going stability studies
}

\author{
Dragana Kafedziska*, Marina M. Micevska, Petranka P. Janoska, Elena Cvetanovska, \\ Mena I. Zdravkovska, Milos Todorovski, Aleksandar Janusevski, \\ Magdalena Blazevska, Hristina Babunovska
}

Alkaloid AD-Skopje,Pharmaceutical, Chemical and Cosmetics Industry, blvd.Aleksandar Makedonski 12, 1000 Skopje, Repablic of Macedonia

\section{Introduction}

In ALKALOID AD Skopje, the stability test of commercial batches of pharmaceuticals is performed with on-going stability studies. Long-term tests are used for these studies, and samples for that purpose are stored, until expire date, in suitable walk-in chambers, under defined storage conditions: temperature $\left(25^{\circ} \mathrm{C} \pm 2^{\circ} \mathrm{C}\right)$ and relative humidity $(60 \% \mathrm{RH} \pm 5 \% \mathrm{RH})(\mathrm{ICH}, 2003)$. The defined storage conditions (temperature and relative humidity) must be maintained within the acceptable limits.

The aim of this work is to estimate the risk of temperature and relative humidity deviation in the walk-in stability chambers, taking measures for its control and reduction.

\section{Materials and methods}

The FMEA (Failure Mode Effects Analysis) method was used to perform this risk assessment (ICH, 2015).

FMEA provides an organized, critical analysis of potential failure modes of the system being defined and identifies associated causes (ICH, 2015). It uses occurrence and detection probabilities in conjunction with severity criteria to develop a risk priority number (RPN) for ranking corrective action considerations. RPN has been calculated on the basis of multiplication of the following indicators: severity, detection and probability.

\section{Results and discussion}

Quality risk management is a systematic process for the assessment, control, communication and review of the risks to the quality of the drug product across the product lifecycle (ICH, 2015; WHO, 2014).

Risk management methodology is based on the following:

\section{Risk assessment}

Risk identification - deviation of the defined storage conditions (temperature and/or relative humidity) is accomplished by monitoring systems. Identification of risk provides the basis for further steps in the quality risk management process.

Risk analysis - it is the qualitative or quantitative process of linking the likelihood of occurrence and severity of harms (ICH, 2015).

\footnotetext{
*dkafedziska@alkaloid.com.mk
} 
Sources of deviation of temperature and/or relative humidity might be:

- failure in the system for maintaining defined conditions;

- failure in the monitoring systems;

- inappropriate managing with walk-in chambers (e.g. inadequate door closing);

- inability to connect the generator to a voltage drop.

Risk evaluation - compares the identified and analyzed risk against given risk criteria. In case the defined conditions cannot be established in acceptable limits within 24 hours, the samples are transferred to another stability chamber with required performances. Corrective actions should be identified and implemented following the investigation to prevent reoccurrence (WHO, 2014).

The value of the RPN was estimated and calculated as 4 .

\section{Risk control}

This phase includes decision making to reduce and/or accept the risk. The purpose of the risk control is to reduce the risk to an acceptable level.

Established measures for risk control are:

- Continuous monitoring of temperature and relative humidity with two independent devices, MOGAL which is an integral part of the chambers and TESTO SAVERIS with its own separated probes and software.

- The values of the two monitoring devices are recorded at precisely determined and continuous time intervals. All records are documented and reviewed constantly.

- Monitoring alarms and alarms check - set points for alarms contain alert and action limits. The alert limits are set to allow preventive actions. These alarms alert relevant and trained persons in the form of SMS and e-mail messages, allowing timely detection of possible deviation.

- All processes related to chamber management, monitoring systems, documentation and recording of the values recorded by the two monitoring devices, calibration of the measuring instruments, chamber mapping are described with appropriate procedures and SOPs.

- Appropriate training should be provided for all staff members involved in the process of stability studies.
Additional risk management measures for risk control that are taken depending on the deviation background are:

- Temperature and relative humidity monitoring records are regularly reviewed and approved to ascertain whether a deviation may have occurred.

- Re-training should be provided in cases when deviation is due to inappropriate management with walk-in chambers.

- Re-calibration of sensors in cases where their validity is suspected.

\section{Risk review}

Once a quality risk management process has been initiated, that process should continue to be utilized for events that might impact the original quality risk management decision. The frequency of any review should be based upon the level of risk. Risk review might include reconsideration of risk acceptance decisions.

\section{Conclusion}

Based on the results of the risk assessment, it was concluded that the risk of temperature and/or relative humidity deviation in the walk-in stability chambers is considered as low. The benefit of this risk assessment is a reduction in the risk of deviation of storage conditions that prevents current stability studies from being compromised.

\section{References}

International Council for Harmonization of Technical Requirements for Pharmaceuticals for Human Use; ICH guideline Q1A(R2) on Stability Testing of new Drug Substances and Products; August, 2003.

International Council for Harmonization of Technical Requirements for Pharmaceuticals for Human Use; ICH guideline Q9 on quality risk management, Q9; September, 2015.

Technical supplement to WHO Technical report series, No. 961, 2011, Annex 9: Model guidance for the storage and transport of time and temperature sensitive pharmaceutical products; Temperature and humidity monitoring system for fixed storage areas, January, 2014. 